\title{
Computational design of peptide vaccine against Acinetobacter baumannii infection using comparative genomic approach
}

\author{
Ajao Abdullahi Taiwo ${ }^{1, *}$, Ajao Jumoke Falilat ${ }^{2}$, Yakubu Sabo Ezemuel ${ }^{3}$ \\ ${ }^{1}$ Department of Science Lab. Tech, Kwara State Polytechnic, Ilorin, Nigeria \\ ${ }^{2}$ Department of Computer, Library and Information Science, Kwara State University, Malete, Nigeria \\ ${ }^{3}$ Department of Microbiology, Ahmadu Bello University, Zaria, Nigeria
}

\section{Email address:}

ajaoabdullahi@yahoo.com (A. A. Taiwo)

\section{To cite this article:}

Ajao Abdullahi Taiwo, Ajao Jumoke Falilat, Yakubu Sabo Ezemuel. Computational Design of Peptide Vaccine against Acinetobacter Baumannii Infection using Comparative Genomic Approach. Computational Biology and Bioinformatics. Vol. 2, No. 1, 2014, pp. 13-18. doi: $10.11648 /$ j.cbb.20140201.13

\begin{abstract}
The bacterial species Acinetobacter baumannii is a major cause of hospital acquired infection throughout the world and it is increasing public health concern. Infection caused by multidrug resistant A. baumannii is currently among the most difficult to treat due to propensity to acquire mobile genetic element. To date there is no vaccine or specific drug available for its treatment, this necessitate the need for the identification of therapeutic target enzyme and vaccine. Pharmacogenomic and computational biology represent an attractive alternative approach for the identification of common drug target and peptide-vaccine candidates in the pathogen. Vaccine designing is shifted from entire pathogen or whole antigen to peptide or epitope based-vaccines that are specific, safe and easy to produce. Comparative genomic approach was used to identify conserved protein signatures among five genomes. Three outer membrane proteins conserved among the genomes with high vaxijen scores were used to produce both B-cell and T-cell mediated immunity. Propred and propred1 were used to predict promiscuos helper T-Lymphocytes (HTL), Cytotoxic T-Lymphocyte (CTL) epitopes and MHCPred for their binding affinity.Three T-cell epitopes derived from identified B-cells bind to maximum number of MHC class I and class II alleles and specifically bind to HLA alleles such as DRB $1 * 0101$ and DRB1*0401.The epitopes are YEKLAAGPS, FYTSQPEDS and YVTGNPLGL with high potential to induce humoral and cell mediated immune responses. These predicted epitopes (small peptide) might be promising candidates for vaccine design against $A$. baumannii infection, though experimental validation.
\end{abstract}

Keywords: Peptide Vaccine, Pharmacogenomic, Cell Mediate Immunity, Drug Resistance, Epitopes

\section{Introduction}

Acinetobacter baumannii is a major cause of hospitalacquired infection worldwide due to its remarkable propensity to rapidly acquire determinants to a wide range of antibacterial agent. Today, some strains of $A$. baumannii have become resistant to almost all currently available antibacterial agents, mostly through the acquisition of plasmids, transposons or integrons $[5,6]$.

Infections caused by $A$. baumannii are associated with adverse clinical outcomes, including high rates of morbidity and mortality, prolonged hospital stay, and substantial health care expenses. Because of the multiple antibiotic resistance exhibited by $A$. baumannii, nosocomial infections caused by this organisms coupled with the fact that those bacteria have a significant capacity for long-term survival in the hospital environment, thus favouring the transmission between patients either via human reservoirs or via inanimate material [7,8]. The management of Acinetobacter baumannii infection has become a public health problem in many countries.

Treatment of infection due to Acinetobacter has become challenging and the need to identify new antimicrobial target is more pressing than ever. The search for new targets or vaccine candidates is of high paramount. To date, there are no vaccines that have been developed for this organism. Bioinformatics-based approach is a novel platform to identify drug targets and vaccines candidates in 
human pathogen. This technique has been successfully used by several authors to identify drug target vaccine candidates. Peptide - based sub-unit vaccine has recently attracted attention in both treating infectious diseases and also for promoting destruction of cancerous cells [9, 10], these type of vaccines are easy to produce and also safe when compared to the usual vaccines like killed vaccines and attenuated vaccine. Present study aimed to use comparative genomic approach to identity epitopes that can produce the $\mathrm{B}$-cell and $\mathrm{T}$-cell mediated immunity to develop peptide-based vaccines against $A$. baumannii.

\section{Material and Methods}

Genome Sequence: Complete genome sequences of $A$. baumannii were obtained from Genebank database. Ftp://ftp.ncbi.nih.nlm.gov/genome under the Genebank accession numbers: NC_005966.gbk, NC_011595gbk, NC_017171gbk, NC_017162gbk and NC_018706gbk. Comparative analysis of genome was carried out using progressive Mauve V. 2.3.1. Homologous proteins coding sequence at the same relative positions in thee genomes were identified and subjected to BLASTp analysis against the $H$. sapiens at an E-value cut off $10^{-3}$. BLAST results with No hits with $H$. sapiens were classified as nonhuman homologues enzymes. All non-human homologues proteins were subjected to the program PSORTb V.3 (http.//www.psort.org/psortb/index.Html) for subcellular localization prediction. All the proteins present in the outer membrane were analyzed using vaxijen $\mathrm{V} 2.0$ antigen predicition server (www. Ddg-pharmfac.net vaxijen). The default parameters (Threshold $=0.4$, ACC Output) were used against bacterial species to check the antigenicity of each full length protein sequence. Proteins having antigenic score $>0.6$ were selected for B-cell epitope prediction.

For prediction of B-cell epitopes each full length protein sequences was subjected to BCPreds analysis. Both BCpred and AAP prediction methods of BCPreds were used to identify common B-cell epitopes (http://ailab .cs.iastate.edu/bcpreds). All prediction Bcell epitopes (20mers) having a BCpreds cut off score > 0.7 was selected.

Selected B-cell epitopes were then subsequently checked for membrane topology using TMHMM V 2 for exo-membrane amino acid sequences (www.cbs.dtu.dik)

Each antigenic B-cell epitope sequences were then analyzed with Propred-1 for MHC Class1 and Propred for MHC Class II epitope prediction using default parameters. Common epitopes for both the MHC classes that also can bind to maximum MHC Alleles were selected and calculated using MHCPred V.2 selecting [DRB 1*0101] and Vaxijen respectively. Epitopes with highest antigenicity and those bind more than $15 \mathrm{MHC}$ molecules comprising of both the MHC class I and II alleles and less than $50 \mathrm{nM} 1 \mathrm{C}_{50}$ scores for DRB1*0101 were selected.

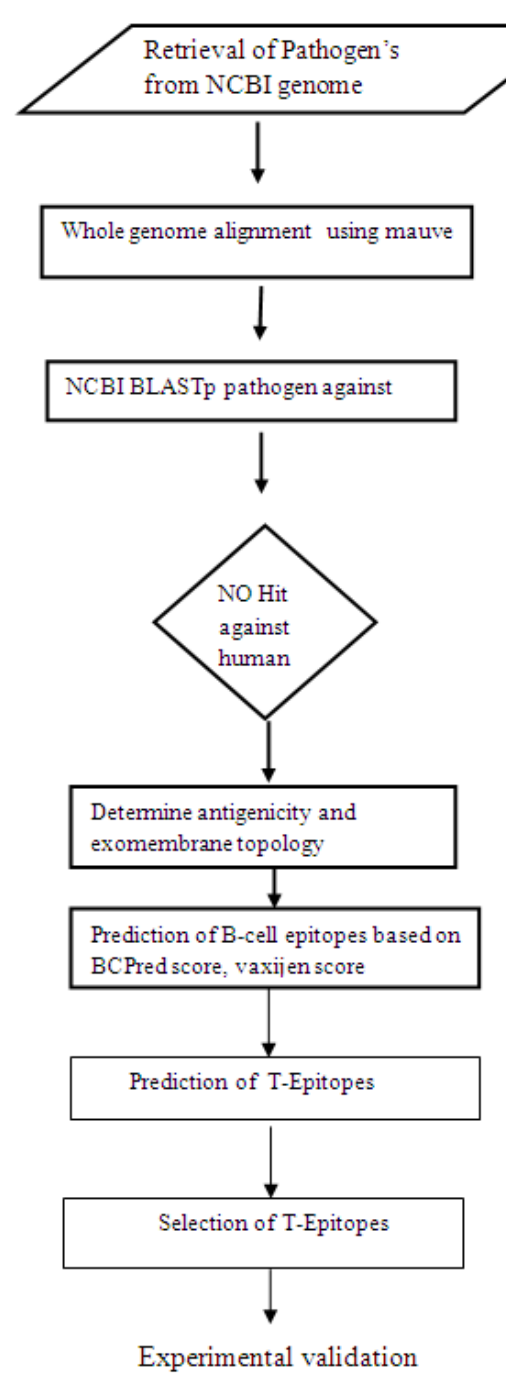

Figure 1. comparative genomic workflow.

\section{Results and Discussions}

The rapid transmission and subsequent infection of patients with multi-drug resistant $A$. baumannii has become a major concern in hospital and other health care facilities . It is known to be difficult to prevent Acinetobacter spp infection in hospital patients because the organisms are ubiquitous in hospital environment and infections caused by multidrug-resistant. A baumannii is currently among the most difficult ones to treat. Therefore, infection control is crucial, particularly given the ability of $A$ baumannii to cause outbreak. Immunization of humans with whole bacteria may not be feasible as it raises various safety concerns due to the presence of lipopolysaccharide (LPS). Availability of genome sequences of pathogens has provided a tremendous amount of information that can be useful in drug target identification and vaccine candidates. Earlier studies have reported either T-cell or B-cell based on epitope designing for a given pathogen [21, 22, 23, 24]. An epitope that can produce both B-cell and T-cell mediated immunity is highly desirable for designing peptide based vaccines. 
The present study used Mauve 2.0 to efficiently construct multiple genome sequence alignment. This tool identified genomic recombination events such as gene loss, duplication, rearrangement and horizontal transfer and homology protein shared by Acinetobacter baumannii Indepth analysis of this genome in the A. baumannii identified 100 proteins conserved in all five A. baumannii genomes. This conserved genomic region unique to $A$. baumannii could not only serve as drug target enzyme, vaccine candidate and also a biomarker for new clinical strains as shown in Figure 2.

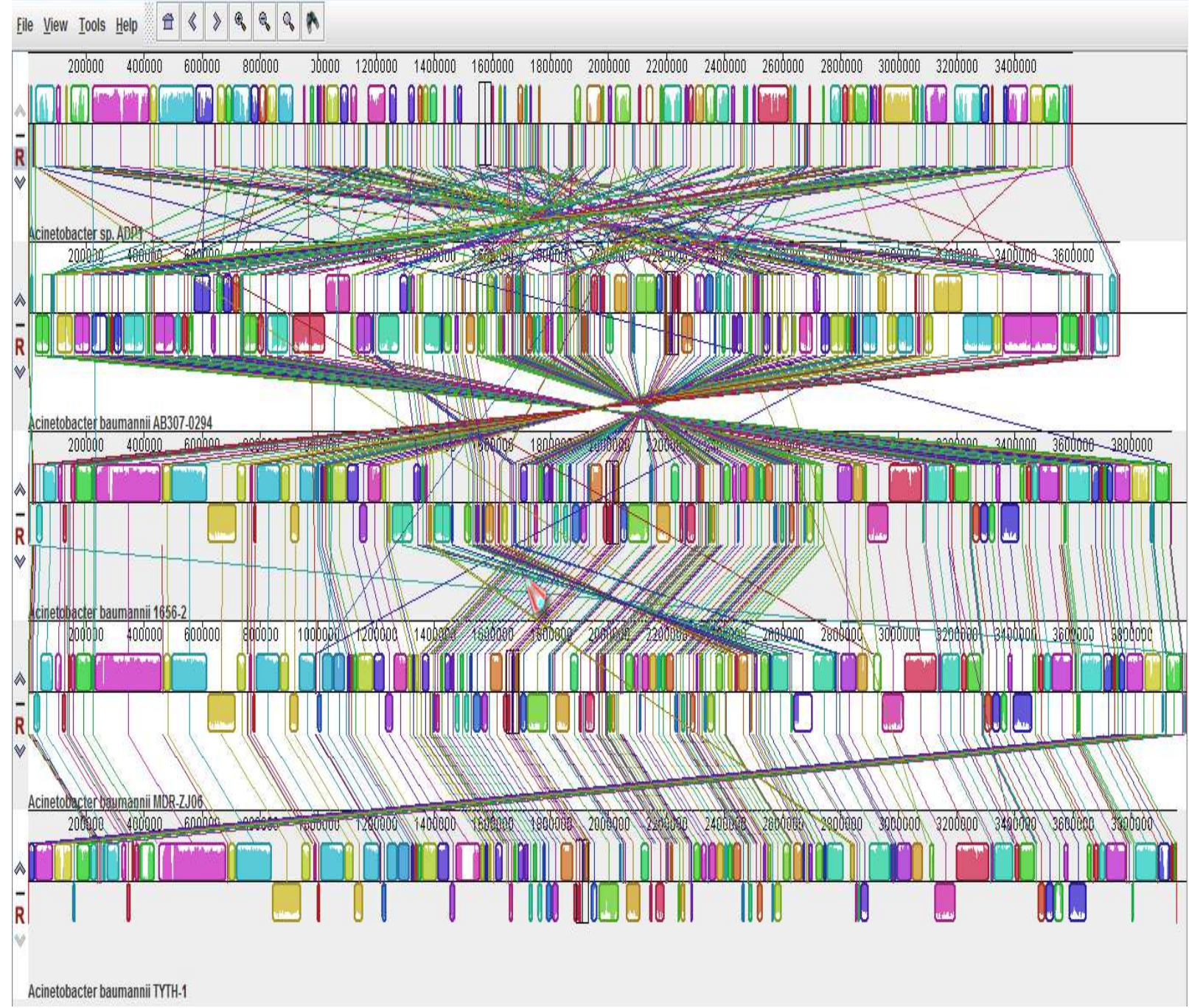

Figure 2. Multigenome comparison between Acinetobacter baumannii strains obtained by use of the Mauve tool.

A definite homology between the host and pathogen protein chosen as drug targets might lead to unwanted cross-reactions and cytotoxicity.

Therefore, enzymes from the A. baumannii that share a similarity with the host proteins were removed to ensure that the targets have nothing in common with the host proteins and thereby eliminating undesired host proteindrug interaction. BLAST $\mathrm{P}_{\mathrm{P}}$ similarity search of all these 100 enzymes at an E-value cutoff of $10^{-3}$ resulted in 52 nonhomologues enzymes of $A$. baumanni out of which 14 were membrane associated non-human homologoues proteins as shown in Table 1.

Table 1. List of Membrane Associated Non-Human Homologous Protein in Acinetobacter baumannii.

\begin{tabular}{|c|c|c|c|c|}
\hline $\mathbf{S} / \mathbf{N}$ & Protein Product & Protein ID & Vaxijen Score & Localization \\
\hline 1 & Drug/Metabolite Transporter & YP_005526256.2 & 0.5695 & Cytoplasmic \\
\hline 2 & Putative Iron-regulated membrane protein & YP_00552625812 & 0.6334 & Cytoplasmic \\
\hline 3 & Outer membrane Receptor Protein & YP_005525884.1 & 0.6079 & Outer membrane \\
\hline 4 & Putative Penicillin Binding protein & YP_005527536.1 & 0.4914 & Extracellular \\
\hline 5 & Glutamate synthase Large chain precursor & YP_005527527.1 & 0.4914 & Extracellular \\
\hline 6 & $\mathrm{~K}+$ Transporter & YP_005527527.1 & 0.4994 & Cytoplasmic membrane \\
\hline 7 & Cation/Multidrug Efflux Pump & YP_005527046.1 & 0.5782 & Cytoplasmic membrane \\
\hline
\end{tabular}




\begin{tabular}{lllll}
\hline S/N & Protein Product & Protein ID & Vaxijen Score & Localization \\
\hline 8 & Preprotein translocase Sub unit sec A & YP_005527240.1 & 0.5414 & Cytoplasmic \\
9 & Organic solvent Tolerance protein OstA & YP_005525712.1 & 0.6282 & Outer membrane \\
10 & DNA Segregation ATPase Ftsk & YP_005524822.1 & 0.5127 & Cytoplasmic Membrane \\
11 & Putative Vanillate .O- Demethylase Oxygenase Subunit & YP_005525005.1 & 0.5462 & Cytoplasmic \\
12 & Outer membrance receptor for Ferrienterochelin and colicins & YP_00552625041.1 & 0.8039 & Outer membrane \\
13 & Solube lytic murein Tansglycosylase & YP-005525200.1 & 0.6476 & Outer membrane \\
14 & Ribonuclease T & YP-005525207.1 & 0.5638 & Cytoplasmic \\
\hline
\end{tabular}

According to who reported that outer membrane Gramnegative pathogenic bacteria has an important role in the interaction with hosts in bacterial pathogenicity, playing a role in adherence, uptake of nutrients from the host, and countering host defense mechanisms. They could be protective antigens because the components of the outer membrane are easily recognized as foreign substances by immunological defense systems of hosts, this informed the reason for the analysis of non-human homologous proteins in A. baumannii for their localization in outer membrane. The PSORTb server was used to identify non-homologous proteins that reside in the outer membrane (Table 2)

Table 2. Selected B-cell epitopes using BCpreds and antigenicity of Protein using BCPred \& Vaxijen score.

\begin{tabular}{llllll}
\hline S/N & Protein & Amino Acid Position & BCpred epitope sequence & BCpred Score & Vaxijen score \\
\hline 1 & Soluble lytic murein transqlycosylase & 433 & PVAVTPAANIKPVRTEPPIS & 0.998 & 0.8442 \\
2 & Outer membrane receptor for & 156 & LEVLRGPAAARYGSGAAGGV & 0.914 & 1.1205 \\
& ferrienterochelin and colicins & & & & \\
& $"$ & 190 & SVEFYTSSQPEDSKEYSSNRV & 1 & 1.6329 \\
3 & Outer membrane receptor protein & 421 & YEDTVDYSPSSEESPGDRYKA & 0.996 & 0.8752 \\
& $"$ & 425 & VDYSPSSESPGDRYKALESD & 1 & 0.8371 \\
4 & Organic solvent tolerance & 250 & DVPVLAVPYFNFPIDDRRTT & 0.904 & 1.3434 \\
& $"$ & 251 & VPVLAVPHFNFPIDDRRTTG & 1 & 1.4695 \\
& $"$ & 272 & LNPQFGFSNDGGIELSVPVY & 1 & 1.3910 \\
& $"$ & 522 & KSVVVPQFTLDTGLNFEREG & 1 & 0.9084 \\
& $"$ & 438 & NYVTGNPLGLQYEFNN OTAY & 0.961 & 0.8247 \\
\hline
\end{tabular}

Four membrane associated proteins were selected to identify epitopes which induce both B-cell and T-cell mediated immunity are known to be good vaccine candidates $[15,10]$ to identify epitopes, full length protein were subjected to B-cell epitopes prediction using BCPreds server. All B-cell epitopes were listed from each protein as shown in (Table 3).

Table 3. Common epitopes that can induce both B-cell and T-cell Mediated Immunity.

\begin{tabular}{|c|c|c|c|c|c|}
\hline Epitopes & Amino acid position & Vaxijen score & $\begin{array}{l}\text { MHCPred DRB } \\
1 * 0101\end{array}$ & $\begin{array}{l}\text { IC } 50 \text { value DRB } \\
1 * 0401\end{array}$ & $\begin{array}{l}\text { Total No of MHC binding } \\
\text { (Propred1 \& Proped) }\end{array}$ \\
\hline IKPVRTEPP & 442 & 1.166 & 184.93 & 622.30 & 14 \\
\hline YEKLAAGPS & 773 & 0.7068 & 0.58 & 537.03 & 15 \\
\hline FYTSQPEDS & 193 & 0.6327 & 2.43 & 399.02 & 17 \\
\hline VDYSPSSES & 425 & 1.0520 & 166.14 & 131.52 & 10 \\
\hline VPVLAVPYE & 251 & 1.8662 & 903.65 & 166.72 & 29 \\
\hline FSNDDGGIEL & 278 & 1.5027 & 91.41 & 862.98 & 24 \\
\hline VVPQFFTLDT & 525 & 1.7078 & 476.43 & 52.36 & 48 \\
\hline YVTGNPLGL & 439 & 1.3056 & 6.82 & 210.86 & 32 \\
\hline
\end{tabular}

Epitopes having BCPreds and vaxijen cutoff value respectively $>0.9$ and $>0.8$ were selected for identification of T-cell epitope. The common epitopes, that can bind both MHC classes and covers maximum (more than 15) MHC alleles, were selected using propred I and propred servers. In this study, eight T-cell epitopes were selected in four selected non-homologous protein associated proteins. The selected epitope were further analyzed for vaxijen score and MHCPred $\mathrm{IC}_{50}$ value MHCPred (DRB1* 0101 alleles) was used to identify common T-cell epitopes which can interact with both the MHC classes with highest number and specifically interact with DRB1* 01010. The T-epitope must interact with HLA DRB1* 0101 alleles and secure $\mathrm{IC}_{50}$ value not more than 50 which will indicate good binders. The predicted output is given in units of $\mathrm{IC}_{50} \mathrm{nM}$. A lower value of Ppeptidee $\mathrm{IC}_{50}$ indicates higher affinity towards MHC Molecules. VPVLAVPYF, VVPQFTLDT, IKPVRTEPP, VDYSPSSES, FSNDGGIEL found to bind total MHC score of 29, 48, 14, 10, 24 respectively inspite of antigenicity score of $>0.6$ but because of its high $\mathrm{IC}_{50}$ value for DRB1* 0101, they may not be considered as good vaccine candidates while the following epitopes can be considered as vaccine candidates: "YEKLAAGPS, FYTSQPEDS, YVTGNPLGL and FSNDGGIEL. 


\section{Conclusion}

This comparative genomic approach successfully identified a conserved genomic region among five genomes of A. baumannii that were able to induce both B-cell and Tcell mediated immune responses, these could serve as diagnostic biomarker and vaccine candidates. The availability of full genome sequences and computer-aided analysis to identify putative antimicrobial drug target has become a new trend in pharmacogenomic. This present finding underscores the utility of large genomic databases for insilico systematic drug target identification in the post genomic era.

The predicted epitopes, YEKLAAGPS, FYTSQPEDS and YVTGNPLGL were antigenic and have much potential to interact with most common human HLA alleles. These might be promising candidates for vaccine design against Acinetobacter baumannii. However, experimental validation is required to ascertain their usefulness.

\section{Acknowledgements}

The authors wish to thank the Tertiary Education Trust Fund (TETFUND) for providing fund and also thank the management of our institutions for creating enabling environment.

\section{References}

[1] Fournier, P.E., Valleneet, D., Barbe, V., Audic, S., Ogata, H. Piorel, I., et al. (2006).Comparative genomics of multidrug resistance in Acinetobaccter baumannii. PLOS Genet., 2:E7. DOI: 10.1371/journal.pgen.0020007

[2] VanLooveren, M. and Goossens, H. (2004) Antimicrobial resistance of Acinetobacter spp. Europe. Clin. Microbiol. infect., 10:684-704.DOI: 10.1111/j.1469-0691.2004.00942.x

[3] Seifert, H., Boullion, B., Schulze, A. and Pulverer, G. (1994) Plasmid DNA Profiles of A.baumannii. Clinical Application in a complex endemic setting. Infect. Control Hosp.Epidemiol., 15:520-528. [PubMed]

[4] Devaud, M., Kayser, F.H., and Bachi, B. (1982). Transposon-mediated multiple antibiotic resistance in Acinetobacter strains. Antimicrob. Agents Chemother., 22:323-329.http://dx.doi.org/10.1128/AAC.22.2.323

[5] Segal, H., Thomas, R., Gay, E.B. (2003). Characterization of class I integron resistance gene cassetes and the identification of a novel IS-like element in A. baumanni. Plasmid, 49:169-178.http://dx.doi.org/10.1016/S0147619X(03)00011-8

[6] Piorel, L. Menuteau, O., Agoli, N., Cattoen, C. and Nordmann, P. (2003). Outbreak of extended-spectrum $\beta$ lactamase VEB-1-Producing Isolates of Acinetobacter baumanni in a French hospital J. Clin Microbiol.,41:3542-7.

[7] Aygun, G., Demirkiran, O., Utku, T., Metc. B.,Urkmez, S., Yilmas,M., Yasar, H., Dikmen, Y. and Ozturk, R. (2002). Environmental contamination during a carbapenem-resistant Acinetobacter baumannii outbreak in an intensive care unit. J Hosp. infect. 52:259-262.
[8] Bergogne-Bérézin, E. and Towner, K. J. (1996) Acinetobacter spp. as nosocomial pathogens: microbiological, clinical, and epidemiological features.Clin Microbiol Rev., 9:148-165. [PubMed]

[9] Florea, L., Halldorsson, B., Kohlbacher O. Schwarts, R., Hoffman, S. et. al., (2003). Epitope prediction algorithms for peptide based vaccine design. Proc. Comput. Soc. Bioinform., 2:17-26.

[10] Prabhavathy, K., Perumal, P. and SundaraBaalaji, N. (2011). In silico identification of B- and T- cell epitopes on OMPLA and $\mathrm{LsC}$ from Salmonella typhi for peptide-based subunit vaccine design. Indian Journal of Biotechnology,10: 440451.

[11] Altshul, S.F., Thomas, L.M, Alejandro, A.S., Jinghu, Z., Zheng, Z., et al., (1997). Gapped Blast and PSI BLAST; a new generation of protein database search programs. Nucleic Acids Res., 17:3389-3402.Doi: 10.1093/nar/25.17.3389.

[12] Gardy, J.L., Laird, M.R., Chen, F. et al., (2005) PSORTb v. 2.0: Expanded prediction of bacterial protein subcellullar localization and insights gained from comparative proteome analysis. Bioinformatics, 21 (5): 617-623.

[13] EL-Manzalawy, Y., Dobbs, D. and Honavar, V. (2008). Predicting linear B-cell epitopes using string kernels. J. Mol Recognit. 21:243-255. DOI: 10.1002/jmr.893.

[14] Chen, J., Liu, H., Yang, J. and Chous, K.C. (2007). Prediction of Linear B-cell, epitopes using amino acid pair antigenicity scale. Amino acids, 33:423-428.DOI: 0.1007/s00726-006-0485-9.

[15] Singh, H. and Raghava, G.P. (2001). Propred: prediction of HLA-DR binding sites. Bioinformatics, 17:1238-1237. Doi: 10.1093/bioinformatics/17.12.1236

[16] Guan,P., Doytchinova,I.A. and Flower,D.R.(2003). MHCPred: a server for quantitative prediction of peptideMHC binding Protein Eng., 16:11-18. DOI: 10.1093/nar/gkg510

[17] Dijkshoorn, L., Nemec, A. and Seifert, H. (2007). An Increasing threat in Hospitals: Multidrug-resistant Acinetobacter baumannii. Nat Rev. Microbiol., 5:939-951. [PubMed] [DOI]

[18] Maragakis, L.L. and Perl, T.M. (2008). A. baumannii: epidemiology, antimicrobial resistance and treatment options. Clin. Infect. Dis., 46:12544-63. DOI: 10.1086/529198.

[19] Federicco Perez et al., (2007). Global challenge of multidrug Resistant Acinetobacter baumannii. American society for microbiology, 51(10):3471-3484 DOI: $10.1128 /$ AAC.01464-06

[20] Wroblewska, M. (2006). Novel therapeautic of multidrugPseudomonas aeruginosa and Acinetobacter spp. Infections: the state of the Art-Arch. Immunol. Ther. Exp. (warsz) 54:113-120. DOI: 10.1007/s00005-006-0012-4

[21] Arockiasamy, A. and Krishnaswamy, S. (1995). Prediction of B-cell epitopes for Salmonella typhi OmpC. J Biosci., 20:235-243. Arevalo-Herrea, M., Valencia, A.Z., Vergara, J., Bonelo, A., Fleischhauer, K., et al.,(2002)Identification of HLA-A2 restricted CD8 (+) T-lymphocyte responses to Plasmodium vivax circum sporozoite protein in individuals naturally exposed to malaria. Parasite immunol.,24:161-169. DOI:10.1046/j.1365-3024.2002.00449.x. 
[22] Sollner, J., Grohmann, R., Rapherger, R., Perco, P., Lukas, A et al., (2008). Analysis and Predicition of protective continuous B-cell epitopes on pathogen proteins. Immunomes Res. 4:1-12. DOI:10.1186/1745-7580-4-1.

[23] Menakha, M. and Manoharan, C. (2011). Computer Aided Drug Design from Immunoinfomatics to Reverse Vacccinology. Advanced Biotech., 10:1444-147.
[24] Neema, M., Karunasagar, I. and Karunsagar., I. (2011). In silico identification and characterization of novel drug targets and outer membrane proteins in the fish pathogen Edwardsilla tarda. Open Access bioinformatics, 3:37-42. DOI: $10.2147 / \mathrm{OAB} . \mathrm{S} 15581$.

[25] Seltmann, G. and Holst, O. (2002).The bacterial cell wall. Heidelberg:Springer Berlin. 